Special issue of the 2nd International Conference on Computational and Experimental Science and Engineering (ICCESEN 2015)

\title{
On an Application of Generalized Semi-Infinite Optimization
}

\begin{abstract}
A. TEZEL ÖZTURAN*
Hacettepe University, Faculty of Science, Department of Mathematics, 06800 Beytepe, Ankara, Turkey

Generalized semi-infinite optimization problems are optimization problems having infinitely many constraints. In addition, the infinite index set depends on the decision variable of optimization. In this study, as an application of generalized semi-infinite optimization problems a type of design centering problems is considered. In a general design centering problem some measure of a parametrized body is maximized under the constraint that parametrized body is inscribed in a fixed body. In this study, diamond cutting problem is considered as a type of design centering problems. Here the aim is to maximize the volume of a round cut diamond from functional approximation of a rough irregularly shaped gemstone. First of all, the problem is converted to a generalized semiinfinite optimization problem, then corresponding first order optimality conditions are obtained. Several numerical examples are presented by solving reformulated Karush-Kuhn-Tucker optimality conditions with semismooth Newton method. The advantage of the method is that a linear system of equations has to be solved in each iteration.
\end{abstract}

DOI: 10.12693/APhysPolA.130.14

PACS/topics: 02.06.-X

\section{Introduction}

The article studies an application of generalized semiinfinite optimization problems (GSIP). The problem is solved by semismooth Newton method. A problem of GSIP can be written as

GSIP : minimize $f(x)$ subject to $x \in M$,

with $M=\left\{x \in R^{n} \mid g(x, y) \leq 0\right.$, for all $\left.y \in Y(x)\right\}$ and $Y(x)=\left\{y \in R^{m} \mid v_{j}(x, y) \leq 0, j \in Q\right\}$.

All defining functions $f, g, v_{j}, j \in Q=\{1, \ldots, q\}$, are assumed to be real-valued and at least twice continuously differentiable on their respective domains. In a GSIP problem, the possibly infinite index set $Y(x)$ is allowed to vary with $x$, but in a standard semi-infinite optimization problem the infinite index set is fixed, that is, we have $Y(x) \equiv Y$. Furthermore, in finite optimization problems, the index set is a finite set $Y=\left\{y^{1}, y^{2}, \ldots, y^{p}\right\}$.

\section{Semismooth optimality conditions for GSIP}

The lower level problem of GSIP is defined by

$$
Q(x): \max _{y \in R^{m}} g(x, y) \text { subject to } v_{j}(x, y) \leq 0, j \in Q \text {. }
$$

The main computational problem in semi-infinite programming is that lower level problem has to be solved to global optimality, even if only a stationary point of the upper level problem is sought. Since we replace lower level problem by its KarushKuhn-Tucker conditions, lower level problem must be convex.

For $\bar{x} \in M$ let $Y_{0}(\bar{x})=\{y \in Y(\bar{x}) \mid g(\bar{x}, y)=0\}$ denote the set of active indices of $\bar{x}$. If $\bar{x} \in M$ is a local minimizer of GSIP at which the Reduction Ansatz without strict complementarity (see [1]) and the Extended Mangasarian-Fromovitz constraint qualification

*e-mail: aozturan@hacettepe.edu.tr hold, then there exist a $p \in\{0, \ldots, n\}$ and multipliers $\bar{\mu}_{i} \geq 0, i \in P=\{1, \ldots, p\}$, such that

$$
\begin{aligned}
& \nabla f(\bar{x})+\sum_{i=1}^{p} \bar{\mu}_{i} \nabla_{x} L\left(\bar{x}, \bar{y}^{i}, \bar{\gamma}^{i}\right)=0, \\
& \bar{\mu}_{i} \geq 0, g\left(\bar{x}, \bar{y}^{i}\right)=0, i \in P,
\end{aligned}
$$

where $L\left(\bar{x}, \bar{y}^{i}, \bar{\gamma}^{i}\right)=g\left(\bar{x}, \bar{y}^{i}\right)-\sum_{j=1}^{q} \bar{\gamma}_{j}^{i} v_{j}\left(\bar{x}, \bar{y}^{i}\right)$.

Next, the upper level first order condition is complemented by a lower level first order condition. In fact, since the active indices $\bar{y}^{i} \in Y_{0}(\bar{x}), i \in P$ are global solutions of $Q(\bar{x})$, under some constraint qualification like Slater's condition in the lower level problem, there exist vectors of Lagrange multipliers $\bar{\gamma}^{i} \in R^{q}$ such that

$$
\begin{aligned}
& \nabla_{y} g\left(\bar{x}, \bar{y}^{i}\right)-\sum_{j=1}^{q} \bar{\gamma}_{j}^{i} \nabla_{y} v_{j}\left(\bar{x}, \bar{y}^{i}\right)=0, i \in P, \\
& \bar{\gamma}_{j}^{i} \geq 0, v_{j}\left(\bar{x}, \bar{y}^{i}\right) \leq 0, \\
& \bar{\gamma}_{j}^{i} v_{j}\left(\bar{x}, \bar{y}^{i}\right)=0, i \in P, \quad j \in Q .
\end{aligned}
$$

Now, with any function $\psi$ of nonlinear complementarity problem, see [2], the solution of upper and lower level first order condition is seen to be equivalent to finding a zero of the following function

$$
T(z)=\left(\begin{array}{c}
\nabla f(x)+\sum_{i=1}^{p} \mu_{i} \nabla_{x} L\left(x, y^{i}, \gamma^{i}\right) \\
\ldots(a=1, \ldots, p) \\
\psi\left(\mu_{a},-g\left(x, y^{a}\right)\right) \\
\ldots(b=1, \ldots, p) \\
\nabla_{y} g\left(x, y^{b}\right)-\sum_{j=1}^{q} \gamma_{j}^{i} \nabla_{y} v_{j}\left(x, y^{b}\right) \\
\ldots(c=1, \ldots, q) \\
\psi\left(\gamma_{b}^{c},-v_{b}\left(x, y^{c}\right)\right)
\end{array}\right)
$$

where $T: R^{N} \rightarrow R^{N}, N=n+(m+q+1) p, x \in R^{n}$, $\mu \in R^{p}, y \in R^{m p}$ and $\gamma \in R^{p q}$. Then $z \in R^{N}$ and $T$ strongly semismooth under our assumptions. 


\subsection{Semismooth Newton method}

In this section, semismooth Newton method is presented to solve $T(z)=0$. Let $\theta(z)=\frac{1}{2} T(z)^{T} T(z)$, then $\nabla \theta(z)=W^{T} T(z)$, where $W \in \partial T(z)$, here $\partial T(z)$ shows generalized Jacobian of $T$ at point $z$. We use the following algorithm from [2].

\section{Algorithm}

Step 1. Let $z_{1} \in R^{N}, 0<\rho<1,0<\sigma<1, \mu>0$, $a>0$ and $k=1$.

Step 2. If $T\left(z^{k}\right)=0$, then stop. Otherwise, let $d^{k}$ be a solution of

$$
T\left(z^{k}\right)+W^{k} d=0,
$$

where $W_{k} \in \partial T\left(z_{k}\right)$. If (8) is not solvable or if

$$
\nabla \theta\left(z^{k}\right)^{T} d^{k}>-\mu\left\|d^{k}\right\|^{a}
$$

set $d^{k}=-\nabla \theta\left(z^{k}\right)$.

Step 3. Find minimum nonnegative integer $m_{k}$ such that

$$
\theta\left(z^{k}+\rho^{m_{k}} d^{k}\right) \leq \theta\left(z^{k}\right)+\sigma \rho^{m_{k}} \nabla \theta\left(z^{k}\right)^{T} d^{k} .
$$

Set $\alpha_{k}=\rho^{m_{k}}$

Step 4. $z^{k+1}=z^{k}+\alpha_{k} d^{k}$ and $k:=k+1$ and go to Step 2.

Under convexity of lower level and conditions for Clarke subdifferential regularity of generalized Jacobian at the solution point (see [1]) it can be shown that every accumulation point of the sequence $z_{k}$ is a solution of $T(z)=0$ and thus is a stationary point of GSIP. Moreover, it is also possible to show that the algorithm is quadratically convergent under Reduction Ansatz without strict complementarity and Robinson condition for upper level (see [1]).

\section{Design centering problems}

In this study, semismooth Newton method is applied to a special case of design centering problems. In a general design centering problem, the aim is to maximize some measure (e.g., volume) of a parametrized body $B(x)$ under the constraint that $B(x)$ is contained in a fixed body $G$. The formulation of a general design centering problem can be written as

$$
\max _{x \in R^{n}} \operatorname{Vol}(B(x)) \text {, such that } B(x) \subset G .
$$

Let $G=\left\{y \in R^{m} \mid g(y) \leq 0\right\}$ be the fixed body called as container, then GSIP formulation of general design centering problem can be written as follows:

$$
\max _{x \in R^{n}} \operatorname{Vol}(B(x)) \text {, such that } g(y) \leq 0 \text {, for all } y \in B(x)
$$

A good example of design centering problem in three dimensions is the gemstone (diamond) cutting problem. In these problems, the optimal prototype gemstone (diamond being cut) is inscribed into the functional approximation of irregularly shaped rough gemstone [3].

The diamond is parametrized by its geometrical position and orientation within the rough stone, as well as by some other shape parameters such as height, radius, or length-width ratio. There are several shapes for diamonds: round cut, princess cut, trillian cut, etc. In this study we consider several functional approximations of simple rough gemstones and a round cut diamond inscribed into those irregularly shaped simple rough stones. For the first time in literature these problems are solved by semismooth Newton method.

\section{Numerical results}

In this study ten functional approximations of simple irregularly shaped rough stones are considered as containers. These stones have approximately the same vol-

\begin{tabular}{|c|c|c|c|c|c|c|c|c|c|}
\hline Stone & 1 & 2 & 3 & 4 & 5 & 6 & & & 10 \\
\hline Planes & 6 & 10 & 7 & 8 & 11 & $5:$ & 7 & & 12 \\
\hline Paraboloids & 0 & 1 & 2 & 0 & 1 & 2 & & 2 & 0 \\
\hline
\end{tabular}
ume. In the following table, sizes of the containers (simple stones) are presented. In rows, the number of planes and number of paraboloids for functional approximation of stones are given.

The round cut simple diamond is described by $B(x)=\left\{y \in R^{3} \mid v(x, y) \leq 0\right\}$, where $v(x, y)=$ $\left(v_{1}(x, y), \ldots, v_{16}(x, y), v_{17}(x, y)\right)$ with $v_{1}, \ldots, v_{8}$ defining the upper planes, $v_{9}, \ldots, v_{16}$ defining lower planes and $v_{17}$ is the capping plane of the diamond.

The algorithm is implemented in Matlab R2009a. Throughout the computational experiments, the parameters used in the algorithm are $\rho=0.5, a=2.1$, $\mu=10^{-8}$ and $\sigma=0.1$. The algorithm is terminated when $\left\|T\left(z_{k}\right)\right\|<10^{-6}$. The columns of Table I are labeled as follows: "Stone" denotes the number of the functional approximation, "Opt. value" is the optimal value for the volume of diamond, $\|T(\bar{z})\|$ is the Euclidean norm of $T(z)$ at the last iteration point, "CPU" is the CPU time for iterations in seconds, and "Iter." is the number of iterations.

TABLE I

Optimization results from semismooth Newton method.

\begin{tabular}{c|c|c|c|c}
\hline \hline Stone & Opt. value & $\|T(\bar{z})\|$ & CPU & Iter. \\
\hline 1 & 1.398 & $7.8144 \times 10^{-7}$ & 0.42 & 5 \\
2 & 2.021 & $2.3685 \times 10^{-6}$ & 0.89 & 12 \\
3 & 1.534 & $4.0327 \times 10^{-7}$ & 0.62 & 7 \\
4 & 1.497 & $3.6852 \times 10^{-8}$ & 0.73 & 8 \\
5 & 2.013 & $5.6391 \times 10^{-7}$ & 0.92 & 13 \\
6 & 1.427 & $3.7624 \times 10^{-8}$ & 0.81 & 9 \\
7 & 1.743 & $7.5329 \times 10^{-7}$ & 0.75 & 7 \\
8 & 1.932 & $6.0237 \times 10^{-8}$ & 0.78 & 8 \\
9 & 1.841 & $5.8472 \times 10^{-6}$ & 0.88 & 10 \\
10 & 1.921 & $8.5312 \times 10^{-8}$ & 0.94 & 11
\end{tabular}

In the numerical examples, Fischer Burmeister NCP function $\psi_{\mathrm{FB}}(a, b)$ is used [4]. 


\section{Conclusions}

In this paper as an application of generalized semiinfinite optimization a type of design centering problem is considered. For the first time in literature the diamond cutting problems are solved by semismooth Newton method. First of all, the problem of volume maximization for diamond is formulated as a generalized semi-infinite optimization problem. Then semismooth first order optimality conditions are obtained. The lower level convexity is satisfied for these type of problems. The semismooth Newton method is used to solve semismooth optimality conditions. Convergence of this method under standard assumptions is shown in [1, 4]. In [3], the GSIP problems, obtained from diamond cutting problems, are solved by using an interior-point method developed by Stein [5]. Compared with the method used in [3], the advantage of our method is that at each iteration, only a system of linear equations is solved to get search direction. The numerical examples show that semismooth Newton method works well for diamond cutting problems.

\section{References}

[1] O. Stein, A. Tezel, SIAM J. Optim. 20, 1052 (2009).

[2] L. Qi, S.-Y. Wu, G. Zhou, J. Global Optim. 27, 215 (2003).

[3] A. Winterfeld, Europ. J. Op. Res. 191, 838 (2008).

[4] O. Stein, A. Tezel, J. Global Optim. 41, 245 (2008).

[5] O. Stein, Bi-level strategies in semi-infinite programming, Kluwer, Boston 2003. 